\title{
Validity and reliability of the Japanese version of the Patient Assessment of Constipation Quality of Life questionnaire
}

\author{
Haruka Nomura • Takeshi Agatsuma • \\ Toshiki Mimura
}

Received: 28 February 2013/ Accepted: 22 April 2013

(C) Springer Japan 2013

\begin{abstract}
Background This study aimed to provide a psychometric evaluation of the Japanese version of the Patient Assessment of Constipation Quality of Life questionnaire (JPACQOL).

Methods Data for scoring were collected prospectively from patients with constipation who visited our center from 2008 to 2010, and analyzed retrospectively. Reliability of the JPAC-QOL was evaluated using Cronbach's alpha to calculate internal consistency, and a test-retest study was performed to evaluate reproducibility. For concurrent validity assessment, the JPAC-QOL scores were compared with Constipation Scoring System (CSS) scores. In assessing responsiveness, the JPAC-QOL scores before and after treatments were compared in patients whose modified CSS (mCSS) scores decreased by $\geq 50 \%$.

Results Internal consistency was assessed in 295 patients (165 women; mean age 67.0 years). Cronbach's alpha was $>0.7$ for the overall score and all four subscales, showing a
\end{abstract}

Electronic supplementary material The online version of this article (doi:10.1007/s00535-013-0825-y) contains supplementary material, which is available to authorized users.

H. Nomura

Department of Nursing, Kochi Medical School, Kochi

University, Kohasu, Oko-cho, Nankoku, Kochi 783-8505, Japan

T. Agatsuma

Department of Environmental Health Sciences, Kochi Medical

School, Kochi University, Kohasu, Oko-cho, Nankoku,

Kochi 783-8505, Japan

T. Mimura $(\square)$

Pelvic Floor Center, Kochi Medical School, Kochi Medical

School Hospital, Kochi University, 185-1 Kohasu, Oko-cho,

Nankoku, Kochi 783-8505, Japan

e-mail: mimura0523@aol.com strong internal consistency. The intraclass correlations for the 145 patients available for the test-retest study were $>0.7$ for the overall score and for all subscales except satisfaction. The JPAC-QOL scores were significantly associated with the CSS scores in 284 patients, demonstrating concurrent validity in all four subscales and the overall score. The mean JPAC-QOL score improved significantly after treatment in the 72 patients whose mCSS scores decreased by $\geq 50 \%$, indicating good responsiveness in all four subscales and in the overall score.

Conclusions Our study data confirmed the validity and reliability of the JPAC-QOL and demonstrated it ready for use in evaluating the symptom-specific QOL in Japanese patients with constipation.

Keywords Patient Assessment of Constipation Quality of Life questionnaire - Constipation · Validity - Reliability · Quality of life

\section{Introduction}

Constipation impairs quality of life (QOL), causing physical and psychological distress, as well as limiting daily activities $[1,2]$. Selecting an optimal therapy and evaluating the efficacy of treatment depends on individual symptoms and QOL being assessed as accurately and objectively as possible. In 2005, Marquis et al. [3] developed and validated the Patient Assessment of Constipation Quality of Life questionnaire (PAC-QOL), in English, to evaluate QOL specifically in patients with constipation, and this questionnaire has since been widely used in clinical studies of constipation. Our institution routinely uses the PAC-QOL to assess QOL in patients with constipation, and the Constipation Scoring System (CSS) [4] to evaluate symptom severity. 
The original PAC-QOL study [3] also evaluated a French and Dutch version of the questionnaire, and when translated into another language, this scoring system's validity and reliability must again be confirmed in the translated language [5]. This study, thus, sought to validate a Japanese version of the PAC-QOL (JPAC-QOL) and to assess its psychometric properties in Japanese patients with constipation.

\section{Methods}

\section{Patients}

Data for scoring by the JPAC-QOL were collected prospectively after the questionnaire had been self-administered by consecutive patients with a chief complaint of constipation presented to the Pelvic Floor Center, Kochi Medical School Hospital, from September 2008 to December 2010. Patient symptoms were also prospectively evaluated with a structured questionnaire that yielded the CSS score. Patients were also evaluated using the JPACQOL and CSS on two other occasions: on the same day as an evacuation proctography and/or colonic transit study performed without any therapy; and after some patients had received treatment for their constipation.

\section{JPAC-QOL (Appendix 1 in ESM)}

The PAC-QOL is a self-administered questionnaire to assess the constipation-specific QOL in patients with constipation. It comprises 28 questions across the following four subscales: physical discomfort (4 items), psychosocial discomfort (8 items), worries/concerns (11 items), and satisfaction (5 items). The overall score and the scores for each subscale were calculated according to the original PAC-QOL paper [3]. The response scale for each item ranges from 0 to 4, with a higher score indicating worse QOL due to constipation. The scale scores were expressed as the average item response within the scale, and; therefore, the overall score and the scores for each subscale also ranged from 0 to 4 . The missing data rules were applied such that the scale scores were calculated based on the average of the non-missing item responses, as long as at least half of the items in the scale were answered. If more than half of the items in the scale were not answered, the scale score was regarded as incomplete.

The English PAC-QOL was translated into Japanese by one of the authors (TM), who is competent in both Japanese and English, and has considerable expertise in the areas of functional bowel disorders [6-8] and psychometric validation studies [9]. Response to items 25-28 concerning satisfaction were reversed from the original PAC-QOL, to appear as "Extremely", "Quite a bit", "Moderately", "A little bit", and "Not at all" in this order from left to right, so that the lower numbers indicated a better QOL. This modification was required because there was a mistake in the original PAC-QOL, in which the responses were defined by lower numbers indicating a worse QOL.

\section{CSS}

The CSS was developed to evaluate symptom severity in patients with constipation [4]. Its reliability was confirmed by demonstrating significant correlations with objective physiological findings of colonic transit studies, anal manometry, cinedefecography, and anal electromyography in patients with constipation. The CSS comprises 8 items describing the following symptoms of constipation: frequency of bowel movements, painful evacuation, incomplete evacuation, abdominal pain, length of time per attempt, assistance for evacuation, unsuccessful attempts at evacuation per $24 \mathrm{~h}$, and duration of constipation. The score for each item ranges from 0 to 4 with the exception of "assistance for defecation", which is 0-2. Consequently, the overall score for CSS ranges from 0 to 30 with a higher score indicating worse constipation symptoms.

To validate responsiveness, a modified CSS (mCSS) score is created to evaluate the symptomatic improvement with treatment. In the mCSS, "duration of constipation" is excluded from the item list because it does not change at all with any treatment; thus, the mCSS score ranges from 0 to 26 .

\section{Validation methods}

The psychometric properties of the JPAC-QOL were determined in terms of reliability, concurrent validity, and responsiveness, as described below. Analysis was conducted for the four subscales and the overall score.

\section{Reliability}

Internal consistency and reproducibility were investigated to evaluate reliability of the JPAC-QOL. Internal consistency examines the complementary nature of items by searching for contradictions and measurement errors. To evaluate internal consistency, Cronbach's alpha was calculated for the overall score and for all four subscales. A high positive value for Cronbach's alpha $(>0.7)$ suggests that the JPAC-QOL is scoring consistently [10].

To evaluate reproducibility, a test-retest study was performed by comparing JPAC-QOL scores obtained at the time of each patient's first visit with those at the second visit, when an evacuation proctography and/or colonic transit study were undertaken without any interventions applied between the two visits. Comparisons were made using intraclass correlation analysis and a high positive correlation coefficient $(>0.7)$ was taken as evidence of reproducibility [11]. 
Validity

To test the concurrent validity, Pearson's correlation coefficients were evaluated between the JPAC-QOL scores and the CSS scores that can evaluate the symptomatic severity of constipation. Both scores were determined from data collected at the patients' first visit.

\section{Responsiveness}

To assess sensitivity in detecting changes in QOL after treatment, the JPAC-QOL responsiveness was evaluated. This involved comparing JPAC-QOL scores obtained at the patients' first visit with those at their final visit in patients whose symptoms of constipation improved significantly after treatment. Significant symptomatic improvement was defined as a decrease of $\geq 50 \%$ in the mCSS.

Furthermore, the effect size was calculated as (the mean score at post-treatment-the mean score at pre-treatment) divided by the standard deviation at pre-treatment. Effect sizes of $0.2,0.5$, and 0.8 are considered small, moderate, and large, respectively [3].

Statistical analysis

Data are expressed as the mean \pm SD. Statistical analyses were performed using SPSS version 15.0 (SPSS Japan Inc., Tokyo, Japan). Cronbach's alpha, intraclass correlation analysis, Pearson's correlation coefficient test, and a paired $t$ test were conducted as appropriate. A $P<0.05$ was considered significant.

Ethics

This study was approved by the ethics committee of Kochi Medical School (approval no: 23-66). Written informed consent was obtained from all patients who participated in this study.

\section{Results}

During the study period, 332 consecutive patients presented at our center with a chief complaint of constipation. Of these, 37 patients were excluded for various reasons as shown in Fig. 1, thus data from 295 patients were analyzed in this study. The characteristics of the final study patients are given in Table 1.

\section{Reliability}

The evaluation of internal consistency in the JPAC-QOL assessment of the 295 patients returned a Cronbach's alpha value of 0.93 for worries/concerns, 0.82 for psychosocial discomfort, 0.75 for both physical discomfort and satisfaction, and 0.94 for the overall score. These results

332 patients with constipation who visited our center from September 2008 to December 2010

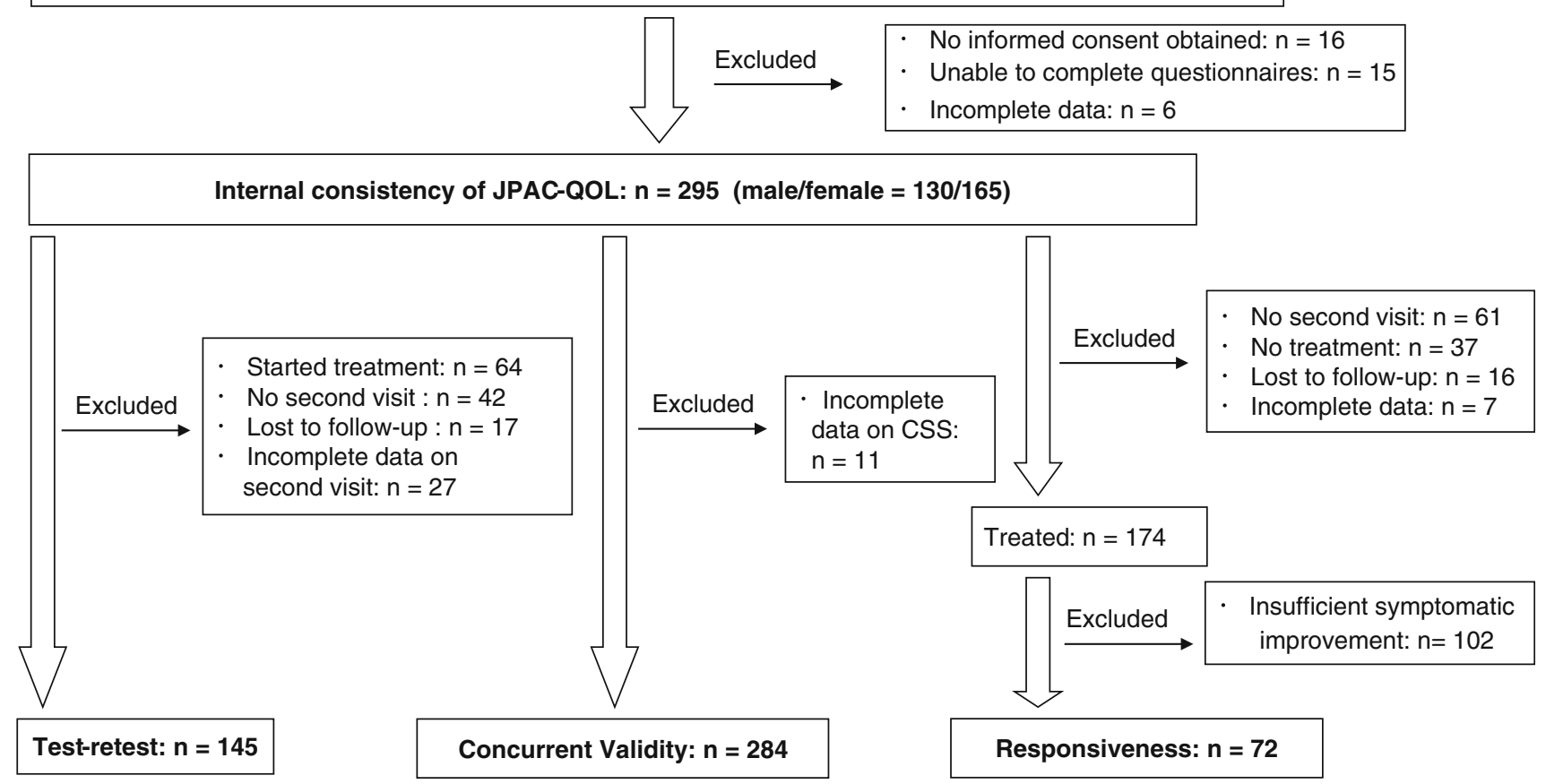

Fig. 1 Flow diagram of patients included in the study and those excluded from analysis for various reasons. JPAC-QOL Japanese version of the Patient Assessment of Constipation Quality of Life questionnaire, CSS constipation scoring system 
Table 1 Characteristics of the 295 patients with constipation

\begin{tabular}{ll}
\hline Age (years) & $67.0 \pm 15.2$ \\
Gender, M/F, $n$ & $130 / 165$ \\
Duration of constipation (years) & $15.1 \pm 14.7$ \\
CSS score $(n=284)^{\mathrm{a}}$ & $13.8 \pm 4.4$ \\
JPAC-QOL score & \\
Physical discomfort & $1.9 \pm 1.0$ \\
Psychosocial discomfort & $1.1 \pm 0.9$ \\
Worries/concern & $2.3 \pm 1.1$ \\
Satisfaction & $3.1 \pm 0.9$ \\
Overall & $2.0 \pm 0.8$ \\
\hline
\end{tabular}

Unless indicated otherwise, data show the mean \pm SD

$J P A C-Q O L$ Japanese version of the Patient Assessment of Constipation Quality of Life questionnaire, CSS constipation scoring system

a 11 patients were excluded due to incomplete CSS score data

indicated that the JPAC-QOL measures consistently for the overall score and across all four subscales.

In the test-retest study, data were available for 145 of the initial 295 patients (49 \%) at the second visit (Fig. 1). The mean interval between the first and second visits was $43.2 \pm 29.6$ days. Intraclass correlation coefficients for the 145 patients are given in Table 2. There was a good correlation between JPAC-QOL scores obtained on the first and second visits for the overall score and across all subscales except for satisfaction.

\section{Concurrent validity}

Data for the assessment of concurrent validity were available in 284 of the 295 patients $(96 \%)$ because the CSS data were incomplete in 11 patients (Fig. 1). The Pearson's correlation coefficients between the JPAC-QOL and the CSS are shown in Fig. 2. The JPAC-QOL was significantly correlated with the CSS in the overall score, as well as in all four subscales, although the correlation coefficients were not so high in the JPAC-QOL scores, ranging between 0.30 and 0.57 .

\section{Responsiveness}

Of the 295 patients in this study, 174 were treated and 72 of these showed significant symptomatic improvement, thus serving as the responsiveness study subjects (Fig. 1). In these 72 patients, the mean overall JPAC-QOL score improved significantly after treatment from 1.9 to 0.5 (Table 3). Similar significant improvements were observed across all four subscales.

The effect sizes were large in all the subscales (ranging from 0.95 to 2.24) and in the overall score (1.84).
Table 2 Reproducibility (test-retest study)

\begin{tabular}{llll}
\hline $\begin{array}{l}\text { Scales } \\
\text { (no. items) }\end{array}$ & $\begin{array}{l}\text { Score at } \\
\text { first visit }\end{array}$ & $\begin{array}{l}\text { Score at } \\
\text { second visit }\end{array}$ & $\begin{array}{l}\text { Intraclass } \\
\text { correlation } \\
\text { coefficient }\end{array}$ \\
\hline Physical discomfort (4) & $2.0 \pm 0.9$ & $1.7 \pm 1.0$ & 0.81 \\
Psychosocial discomfort (8) & $1.2 \pm 0.8$ & $1.0 \pm 0.9$ & 0.86 \\
Worries/concerns (11) & $2.3 \pm 1.0$ & $1.9 \pm 1.1$ & 0.80 \\
Satisfaction (5) & $3.1 \pm 0.9$ & $2.7 \pm 1.0$ & 0.46 \\
Overall (28) & $2.1 \pm 0.7$ & $1.7 \pm 0.8$ & 0.84
\end{tabular}

Data are the mean $\pm \mathrm{SD}$

\section{Discussion}

The present study provides sufficient evidence to support the validity and reliability of the JPAC-QOL for assessing QOL in patients with constipation. The PAC-QOL remains to be formally validated in many of the other languages for which it is now available [12]. In the original English study, French and Dutch versions of the PAC-QOL were administered in France $(n=30)$, the Netherlands $(n=33)$, Belgium $(n=20)$, and Canada $(n=55)$, with demonstrated internal consistency, reliability, and reproducibility [3]. However, these other validation studies involved low patient numbers compared with the original English study $(n=223)$ and our Japanese study $(n=295)$, and the full data and results were not presented. To the best of our knowledge, this is the first study that has formally validated another language version of the original English PAC-QOL.

We demonstrated strong internal consistency for the overall JPAC-QOL score and across all four subscales in our study patients. In particular, Cronbach's alpha coefficients in the overall score and in the worries/concerns subscale were above 0.9 , indicating a high level of internal consistency.

The overall score and three of the four subscales (with the exception of satisfaction) showed good reproducibility, despite the relatively long mean interval of $43.2 \pm$ 29.6 days between the first and second visits. While this long interval was due, in part, to the retrospective nature of our study, it is more likely to reflect the actual situation of clinical practice than a shorter interval of 2 weeks, which was used in the prospective study of the original PAC-QOL [3]. A short interval could overestimate the test-retest reliability because at the time of the second test, patients may still recall what they answered in the first test.

The fairly low intraclass correlation coefficient of 0.46 for the satisfaction subscale obtained in the present study is consistent with that reported in the original study (0.66). One possible explanation for this phenomenon is that the feelings of satisfaction might have improved after the first visit, during which patients could discuss their perceived 
Fig. 2 Concurrent validity of the JPAC-QOL. Pearson's correlation coefficients between the CSS score and the overall JPAC-QOL score (a), physical discomfort (b), psychosocial discomfort (c), worries/concerns (d) and satisfaction (e)
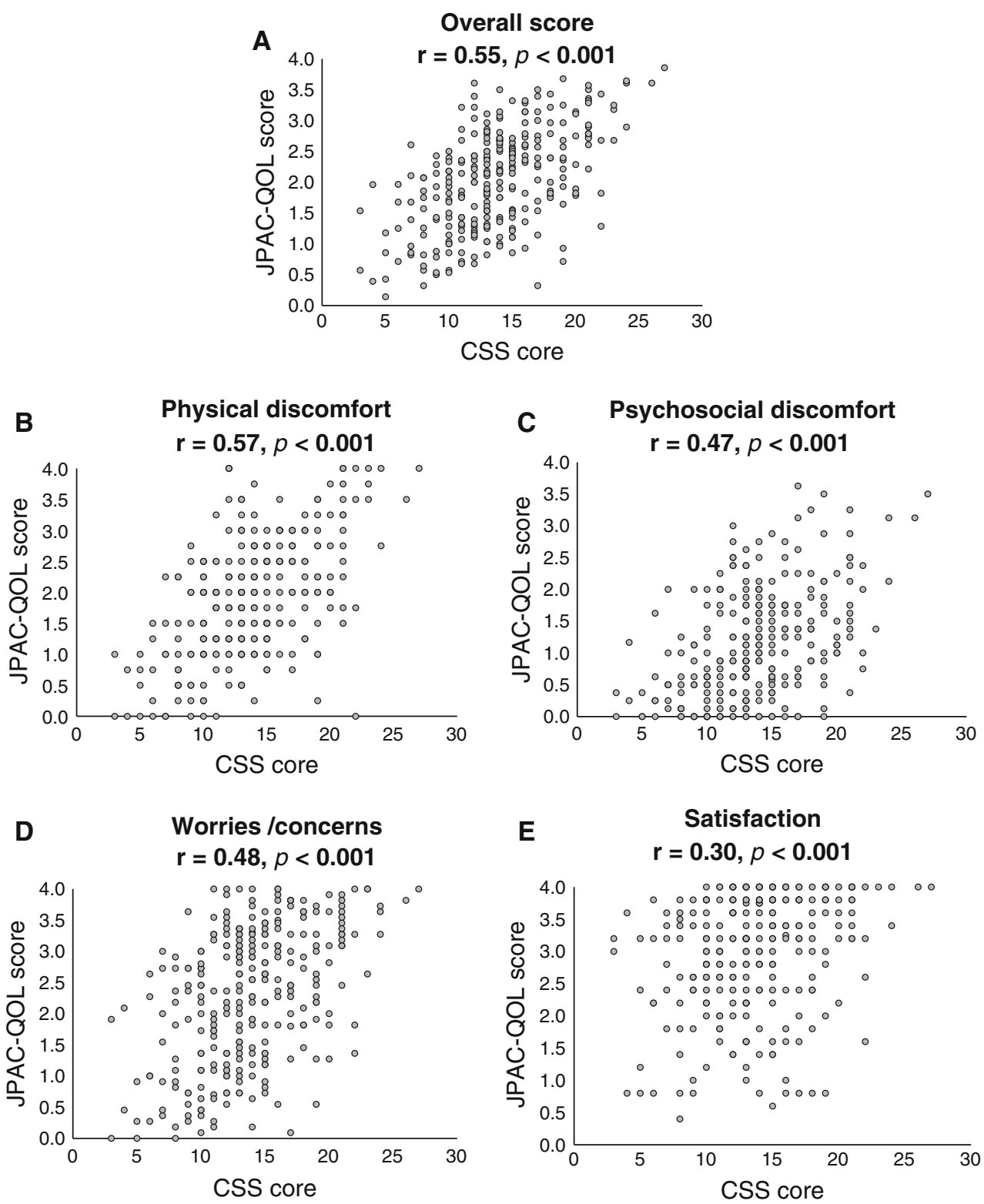

Table 3 Responsiveness

\begin{tabular}{lccccc}
\hline Scale (no. items) & \multicolumn{1}{l}{ Score } & & Mean change $^{\mathrm{a}}$ & Effect size $^{\mathrm{b}}$ & $P$ value $^{(\mathrm{paired} t \text { test) }}$ \\
\cline { 2 - 5 } & Pre-treatment & Post-treatment & & & $<0.001$ \\
\hline mCSS & $12.8 \pm 4.0$ & $4.2 \pm 2.0$ & $-8.6 \pm 2.0$ & 2.16 & $<0.001$ \\
Physical discomfort (4) & $1.8 \pm 1.0$ & $0.4 \pm 0.5$ & $-1.4 \pm 0.5$ & 1.43 & $<0.001$ \\
Psychosocial discomfort (8) & $1.0 \pm 0.9$ & $0.2 \pm 0.3$ & $-0.8 \pm 0.6$ & 0.95 & $<0.001$ \\
Worries/concerns (11) & $2.1 \pm 1.1$ & $0.4 \pm 0.6$ & $-1.7 \pm 0.4$ & 1.56 & $<0.001$ \\
Satisfaction (5) & $3.0 \pm 0.9$ & $1.0 \pm 0.8$ & $-2.0 \pm 0.1$ & 2.24 & $<0.001$ \\
Overall (28) & $1.9 \pm 0.8$ & $0.5 \pm 0.5$ & $-1.5 \pm 0.3$ & 1.84 & \\
\hline
\end{tabular}

Data are the mean \pm SD

${ }^{a}$ Change was computed as the post-treatment score minus the pre-treatment score. Negative change scores indicate QOL improvement

${ }^{\mathrm{b}}$ Effect size $=($ mean at post-treatment - mean at pre-treatment)/SD at pre-treatment. The absolute value is reported in the table 
annoying symptoms of constipation with their doctor. Such a discussion might induce an increase in their feelings of satisfaction at the time of the second visit, even though no actual therapy was performed between the two visits. Moreover, the difference in the mean satisfaction scores between the first and second visits was 0.4 , which is not a clinically significant difference according to some authors. In an evaluation study of the PAC-QOL psychometric properties, Dubois et al. [13] validated a 1-point improvement in the PAC-QOL score as a relevant definition of significant response for treatment. Furthermore, in the original PAC-QOL study, Marquis et al. [3] recommended a 0.5 -point change as the minimum important difference in overall score on the basis of their effect size estimates. These previous two reports imply that a difference of $<1$ or 0.5 points, respectively, is clinically meaningless, and, therefore, that reasonable reproducibility was confirmed in the present study for the subscale of satisfaction, in which the difference in the mean scores between the two visits was 0.4 .

Regarding concurrent validity, there is no gold standard constipation-specific QOL questionnaire that can be compared with the PAC-QOL. Even in the original English study, the PAC-QOL scores were compared with the "clinical severity" to assess cross-sectional validity [3]. The "clinical severity" included the patient-reported number of complete evacuations in the previous week, abdominal pain, global rating of constipation, and investigator-reported global rating of constipation. In the present study, we determined the concurrent validity of the JPACQOL by comparison with the CCS score, which is an established and useful indicator of symptomatic severity of constipation [4]. There was a significant correlation between the CSS scores and the JPAC-QOL scores, confirming the concurrent validity of the JPAC-QOL for constipation symptoms.

Good responsiveness was confirmed in the present study, with the JPAC-QOL scores having increased significantly in accordance with the marked symptomatic improvement. The differences in the scores before and after treatment were more than 1 point, which can be regarded as clinically meaningful [13], in the overall score and all the subscales except for psychosocial discomfort. Similar findings were observed in the original English study, which reported the greatest improvement in satisfaction $(-1.96)$ and the least improvement in psychosocial discomfort $(-0.56)$ [3]. Together, these findings indicate that psychosocial discomfort may be the most difficult to alleviate, whilst the satisfaction feelings may be relatively easy to improve.

The results of the effect sizes in the present study were comparable with those reported in the original PAC-QOL paper [3], which reported the smallest effect size in psychosocial discomfort (0.73) and the largest in satisfaction
(3.41), with the overall effect size being 1.77; these were comparable with the results of the current study.

There were three major limitations in the present study. First, it is a retrospective analysis that resulted in many dropouts because of incomplete data and/or the initiation of treatment prior to the retest. However, most of the 295 study patients completed all of the 28 questions in the JPAC-QOL, as follows: 281 patients $(95 \%)$ in the internal consistency evaluation; 135 (93\%) in the test-retest; $272(96 \%)$ in the concurrent validity; and, 70 (97\%) in the responsiveness assessment. Second, a formal linguistic validation was not performed using a translation-back translation method or a linguistic consensus board. However, in translating the original English PAC-QOL into Japanese, special care was taken to consider cultural and lifestyle differences between the two linguistic societies. Such consideration was also taken in our previous study in validating the Japanese version of the fecal incontinence quality of life scale, and some linguistic modifications were actually made [9]. In the original PAC-QOL, however, no questions seemed unique to an English-speaking society or a certain ethnicity, and; therefore, no linguistic modification was necessary to adapt the English version to Japanese patients. Third, the patients in this study may be different from those with constipation in the general population, because our institute is a tertiary referral center, with numerous referrals for refractory patients. The subjects in our study were relatively old and almost half were male. There were few young women, most likely because young women with slow transit constipation can usually be successfully treated with over the counter medication or by the general practitioner. The results of this study, therefore, might not be applicable to constipated patients in the general population.

The PAC-QOL is the best symptom-specific questionnaire available at present and deserves to be called the gold standard for the evaluation of the symptom-specific QOL in patients with constipation because it has been validated in English, French, and Dutch (albeit inadequately in the latter two). Moreover, the PAC-QOL has been used more and more frequently in many high-quality studies [14]. Currently, no constipation-specific QOL questionnaire is available for the Japanese language that can be used for international comparison. The availability of the PACQOL in various languages would make it possible and easy to compare international studies on constipation, and enable us to conduct international multicenter studies in several languages. We hope that the PAC-QOL is translated, with validation, in more languages. Meanwhile, the JPAC-QOL will be further evaluated in other samples of Japanese patients with constipation.

Acknowledgments The authors thank Dr. Chizu Mimura for her assistance with the statistical analyses. 
Conflict of interest The authors declare that they have no conflict of interest.

\section{References}

1. Irvine EJ, Ferrazzi S, Pare P, Thompson WG, Rance L. Healthrelated quality of life in functional GI disorders: focus on constipation and resource utilization. Am J Gastroenterol. 2002;97: 1986-93.

2. Wald A, Scarpignato C, Kamm MA, Mueller-Lissner S, Helfrich I, Schuijt C, et al. The burden of constipation on quality of life: results of a multinational survey. Aliment Pharmacol Ther. 2007;26:227-36.

3. Marquis P, De La Loge C, Dubois D, McDermott A, Chassany O. Development and validation of the patient assessment of constipation quality of life questionnaire. Scand J Gastroenterol. 2005;40:540-51.

4. Agachan F, Chen T, Pfeifer J, Reissman P, Wexner SD. A constipation scoring system to simplify evaluation and management of constipated patients. Dis Colon Rectum. 1996;39:681-5.

5. Hashimoto H, Shiokawa H, Funahashi K, Saito N, Sawada T, Shirouzu A, et al. Development and validation of a modified fecal incontinence quality of life scale for Japanese patients after intersphincteric resection for very low rectal cancer. J Gastroenterol. 2010;73:928-35.

6. Mimura T, Roy AJ, Storrie JB, Kamm MA. Treatment of impaired defecation associated with rectocele by behavioral retraining (biofeedback). Dis Colon Rectum. 2000;43:1267-72.
7. Mimura T, Nicholls T, Storrie JB, Kamm MA. Treatment of constipation in adults associated with idiopathic megarectum by behavioural retraining including biofeedback. Colorectal Dis. 2002;4:477-82.

8. Mimura T, Kaminishi M, Kamm MA. Diagnostic evaluation of patients with faecal incontinence at a specialist institution. Dig Surg. 2004;21:235-41.

9. Ogata H, Mimura T, Hanazaki K. Validation study of the Japanese version of the fecal incontinence quality of life scale. Colorectal Dis. 2011;14:194-9.

10. Cronbach LJ. Coefficient alpha and the internal structure of tests. Psychometrica. 1951;16:297-334.

11. Chassany O, Sagnier P, Marquis P, Fullerton S, Aaronson N. Patient-reported outcomes: the example of health-Related quality of life-a European guidance document for the improved integration of health-related quality of life assessment in the drug regulatory process. Drug Inf J. 2002;36:209-38.

12. Janssen Global Services. LLC USA and MAPI values. Patient assessment of constipation quality of life questionnaire (PACQOL). http://www.proqolid.org/instruments/patient_assessment_ of_constipation_quality_of_life_questionnaire_pac_qol?fromSearch =yes\&text=yes (2013). Accessed 1 March 2013.

13. Dubois D, Gilet H, Viala-Danten M, Tack J. Psychometric performance and clinical meaningfulness of the patient assessment of constipation-quality of life questionnaire in prucalopride (RESOLOR) trials for chronic constipation. Neurogastroenterol Motil. 2010;22:e54-63.

14. Mueller-Lissner S, Kamm MA, Wald A, Hinkel U, Koehler U, Richter E, et al. Multicenter, 4-week, double-blind, randomized, placebo-controlled trial of sodium picosulfate in patients with chronic constipation. Am J Gastroenterol. 2010;105:897-903. 